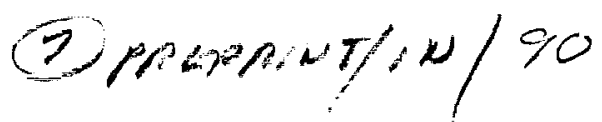

\section{Could the unidentified EGRET sources in the Gould Belt be of pulsar origin?}

\author{
Bing Zhang, ${ }^{*}, 1$ \& Alice K. Harding* \\ *NASA Goddard Space Flight Center, Greenbelt MD 20771 USA \\ ${ }^{\dagger}$ National Research Council Research Associate Fellow
}

\begin{abstract}
We explore the possibility, within the polar cap model, that at least some of the recently identified new population of EGRET sources in the Gould Belt sources are $\gamma$-ray pulsars, viewed at large angles to the magnetic axis, such that the line-of-sight misses both the bright gamma-ray double cone and the radio beam. We estimate that the detectability of such off-beam gamma-ray sources is about a factor of 4-5 times higher than that of the on-beam sources. The off-beam $\gamma$-ray pulsars also have a faint, soft nature, which is consistent with the observed properties of the Gould Belt sources. We conclude that at least some of the middle latitude sources might be such off-beam gamma-ray pulsars if they are indeed in the Gould Belt.
\end{abstract}

\section{INTRODUCTION}

The origin of the 170 unidentified gamma-ray point sources detected by the Energetic Gamma-Ray Experiment Telescope (EGRET) on board the Compton Gamma-Ray Observatory (CGRO) throughout its mission (Hartman et al. 1999) is one of the most intriguing questions of high-energy astrophysics. Recently, Gehrels et al. (2000) studied the spatial and flux distribution of the 120 steady sources in the third EGRET catalog and found that they divide into two groups: higher flux sources distributed along the galactic plane with latitudes less than $5^{\circ}$, and a new population of lower flux sources at mid-latitude. The two groups also show significant differences in their spectra, with the low-latitude sources having average photon spectral index of $2.18 \pm 0.04$ and the mid-latitude sources having average photon index of $2.49 \pm 0.04$. None of these sources have known counterparts at other wavelengths. Grenier \& Perrot (1999) had found that some of the unidentified sources are significantly correlated with the Gould Belt of massive stars, an expanding disk of gas and young stars, most with ages less than 30 million years, surrounding the Sun, at 100-300 pc. They suggested that the Gould Belt $\gamma$-ray sources could be young pulsars, formed in supernova explosions of massive stars of

1) Current address: Astronomy \& Astrophysics Department, Pennsylvania State University, University Park, PA 16802 
the belt. However, the relatively soft spectra and luminosities of these sources are not characteristic of the known $\gamma$-ray pulsars detected by EGRET. Here, we show that many of the Gould-Belt $\gamma$-ray sources could be pulsars if their emission is seen at large angles to their magnetic axes, such that we are missing the bright, hard $\gamma$-ray beams but detecting only the off-beam emission.

\section{OFF-BEAM PULSAR EMISSION}

According to the polar cap model (e.g. Daugherty \& Harding 1996 [DH96]), $\gamma$ ray emission occurs throughout the entire pulse phase. A synchrotron-pair cascade at low altitude radiates the hard on-beam emission in a hollow cone centered on the magnetic pole to produce the bright double-peaked pulses. Primary electrons continue to radiate curvature emission on open field lines to high altitudes beyond the cascade region, producing a lower level of softer off-beam emission. Due to the flaring of the dipole field lines, this emission may be seen over a large solid angle, far exceeding that of the main beams.

The basic geometry of polar cap emission, projected onto the stellar surface, is shown in Figure 1. The main beam of the $\gamma$-ray cascade emission zone is located along the edge of the hollow cone with opening angle $\theta_{\gamma}$, centered on the magnetic axis, denoted by the dotted circle labeled $\gamma$. The altitude of this zone is about $1-2$ stellar radii above the stellar surface (DH96). The off-beam $\gamma$-ray emission zone is located at altitudes above the cascade emission zone, from several stellar radii to the light cylinder. Off-beam emission visible to EGRET above $100 \mathrm{MeV}$ is shown as a shaded region. In Fig. 1, the possible location of radio conal emission with opening angle $\theta_{R}$ is shown as a dashed circle labeled $R$. The circles centered on the rotation axis, labeled $\zeta_{1}$ and $\zeta_{2}$, denote the sweep in pulse phase of different observer lines-of-sight viewing on-beam and off-beam $\gamma$-ray emission respectively.

The maximum height at which EGRET-band emission ( $>100 \mathrm{MeV}$ ) can be produced may be defined as the radius $r_{O B}(\max )$ along the last open field line where the curvature radiation photon energy $E_{0}=100 \mathrm{MeV}$. The typical curvature photon energy is $E_{0}=(3 / 2)\left(\gamma^{3} \hbar c / \rho\right)=322 \mathrm{MeV} \gamma_{7}^{3} P^{-1 / 2}(r / R)^{-1 / 2}$ for dipolar field configuration, where $\gamma_{7} \equiv \gamma / 10^{7}$ is the primary particle Lorentz factor, $\rho$ is the magnetic field radius of curvature, $P$ is the pulsar period and $r$ and $R$ are the radius of the emission point and stellar radius, respectively. Using the expression for the Lorentz factor of a particle traveling along an open dipole field line and subject only to curvature radiation losses is (Harding 1981), Harding \& Zhang $(2000, \mathrm{HZ})$ show that for a typical pulsar, $P=0.3 \mathrm{~s}, \gamma_{0}=2 \times 10^{7}$ and $R_{0}=2 R$, $r_{\mathrm{OB}}(\max )=48 R$.

$\mathrm{HZ}$ show that the off-beam emission detectable to EGRET above $100 \mathrm{MeV}$ will have a luminosity a factor of $\sim 80$ times smaller than the on-beam luminosity. Even so, EGRET can detect off-beam pulsars out to a distance of $d_{\mathrm{lim}} \simeq 260 \mathrm{pc}\left(\Omega_{\mathrm{OB}}\right)^{-1 / 2}$, most of the way through the Gould Belt for a large off-beam solid angle, $\Omega_{\mathrm{OB}}$. If EGRET detects all on-beam and most off-beam pulsars in the Gould Belt, then a 


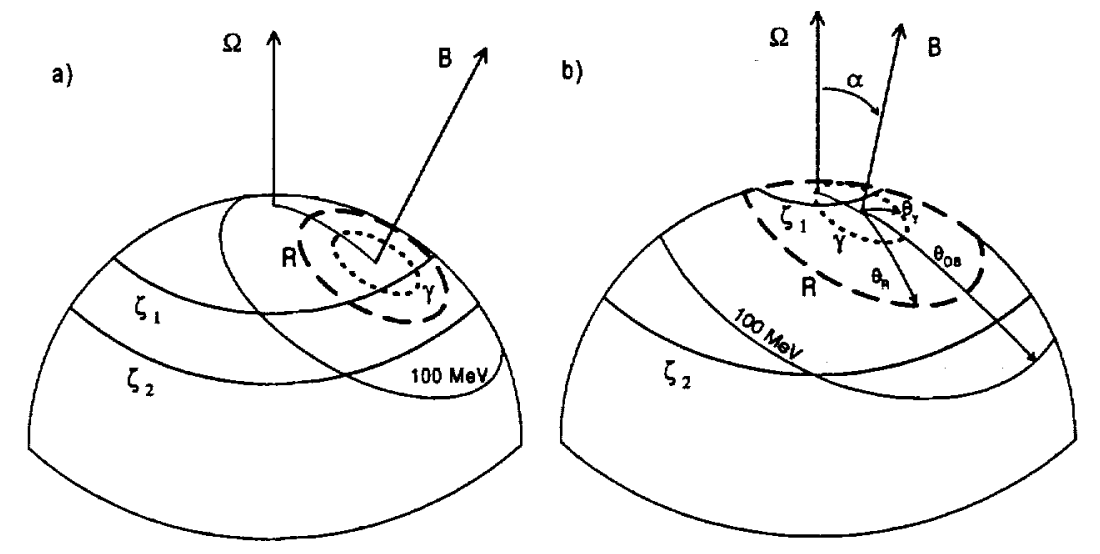

FIGURE 1. The geometry of the polar cap model projected onto the stellar surface. The line-of-sight $\zeta_{2}$ misses both the main gamma-ray beam and the radio emission beam, and we identify such a configuration as the off-beam pulsar candidates for the Gould Belt sources. (a) $\alpha$ not small, $\zeta_{1}$ gives a typical Vela-type emission configuration; (b) $\alpha$ is small, $\zeta_{1}$ gives a typical Geminga-type emission configuration.

larger number of off-beam pulsars should be detectable due to their larger emission solid angle. The relative opening angle, $\theta_{\mathrm{OB}} \sim\left(\Omega r_{\mathrm{OB}}(\max ) / c\right)^{1 / 2}$, of the "100 $\mathrm{MeV}$ cone" with respect to the on-beam $\gamma$-ray cone, $\theta_{\gamma} \sim\left(\Omega r_{\gamma} / c\right)^{1 / 2}$, is: $\theta_{\mathrm{OB}} / \theta_{\gamma} \simeq\left(r_{\mathrm{OB}}(\max ) / r_{\gamma}\right)^{1 / 2}$. Assuming a random distribution of $\alpha$, the relative detectability, $D$, of the off-beam pulsars and the gamma-radio plus Geminga-like pulsars is (Emmering \& Chevalier 1989):

$$
D=\frac{\left(1-\cos \theta_{\mathrm{OB}}\right)+\left(\pi / 2-\theta_{\mathrm{OB}}\right) \sin \theta_{\mathrm{OB}}}{\left(1-\cos \theta_{\gamma}\right)+\left(\pi / 2-\theta_{\gamma}\right) \sin \theta_{\gamma}}-1
$$

Given typical values $P=0.3 \mathrm{~s}, r_{\gamma}=2$, and assuming emission along the "last open field line", one gets $\theta_{\mathrm{OB}} / \theta_{\gamma} \sim 5$ and $D \sim 4$ according to eq.[1]. If we regard Geminga and PSR $0656+14$ (the strongest gamma ray pulsar candidate) as the on-beam gamma-ray pulsars in the Gould Belt, we expect that there might be 8-10 off-beam gamma-ray pulsars, a sizable portion of the 20-40 Gould Belt unidentified EGRET sources (Grenier et al. 2000). The above estimate suggests that at least some of the Gould Belt sources could be off-beam $\gamma$-ray pulsars.

Polar cap cascade simulations show that the spectrum of off-beam emission is softer that that of the on-beam emission. A simulation run for the parameters of the Vela pulsar, using the cascade code developed by DH96, give the brightness and model spectra of $\gamma$-ray emission as a function of $\zeta-\alpha$ is shown in Figure 2. As $\zeta-\alpha$ increases, the spectrum above $100 \mathrm{MeV}$ becomes increasingly softer because the visible emission comes from electrons of decreasing energy at higher altitudes. 


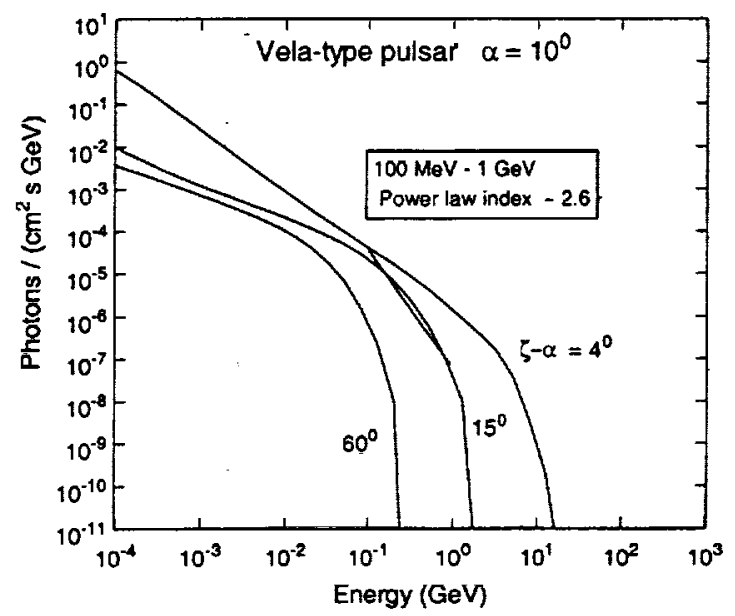

FIGURE 2. Model cascade spectra for different observed lines of sight, $\zeta$ 's. Typical Vela-type pulsar parameters, $P=0.1 \mathrm{~s}, B=4 \times 10^{12} \mathrm{G}$ and $\alpha=10^{0}$ are adopted. The $\zeta-\alpha=4^{0}$ curve is for a typical on-beam pulsar, and the $\zeta-\alpha=15^{\circ}$ and $\zeta-\alpha=60^{\circ}$ curves are off-beam pulsar spectra. A power law fit of the $\zeta-\alpha=15^{\circ}$ spectrum from $100 \mathrm{MeV}$ to $1 \mathrm{GeV}$ gives a spectral index $2.65 \pm 0.57$, consistent with the values observed from the unidentified "Gould Belt sources".

\section{CONCLUSION}

With the polar cap cascade geometry inferred from the known gamma-ray pulsars, we find that there is a large phase space for which the line-of-sight misses both the main gamma-ray beam and possibly the radio beam, but still cuts across a much broader, fainter gamma-ray beam produced by the curvature-radiation cooling of the primary particles. We identify such off-beam $\gamma$-ray pulsars as candidates for the new population of the unidentified EGRET sources associated with the Gould Belt. The off-beam polar cap cascade emission exhibits the low luminosity and soft spectrum in the EGRET band that is characteristic of the observed Gould Belt sources. GLAST will be able to detect pulsed emission from these sources, and the pulse shape should be a broad single-peaked profile.

\section{REFERENCES}

1. Daugherty, J. K., \& Harding A. K.: Ap. J., 458, 278 (1996).

2. Emmering, R. T., \& Chevalier, R. A., ApJ, 345, 931 (1989).

3. Gehrels, N, et al., Nature, $404,363(2000)$.

4. Grenier, I. A. et al., $A \mathscr{E} A$, submitted (2000).

5. Grenier, I. A., \& Perrot, C. , Proc. XXVI ICRC Salt Lake City, 3, 476 (1999).

6. Harding, A. K. , ApJ, 245, 267 (1981).

7. Harding, A. K. \& Zhang, B. , ApJ Letters, submitted (2000).

8. Hartman, R. et al., ApJ Supp., 123, 79 (1999).

9. Zhang, B. \& Harding, A. K. , ApJ, 535, L51 (2000). 